\title{
Triggering final follicular maturation: hCG, GnRH-agonist, or both, when and to whom?
}

\author{
Raoul Orvieto ${ }^{1}$
}

Received: 29 May 2017 / Accepted: 13 June 2017 / Published online: 27 June 2017

(C) Springer Science+Business Media, LLC 2017

\section{Dear Editor,}

We read with interest the study by Elias et al. who retrospectively investigated the utility of a combined GnRH-agonist (GnRHa) and human chorionic gonadotropin (hCG) trigger ("dual trigger") in improving ICSI cycle outcomes in patients with poor fertilization history after standard hCG trigger in prior ICSI cycles [1]. "Dual trigger" was found to increase oocyte maturity, fertilization, clinical pregnancy, and livebirth rates in patients with a history of poor fertilization after standard hCG trigger alone.

While they thoroughly covered the conceptual and practical aspects of the "dual trigger," we still would like to highlight several points that went unnoticed. Nowadays, different modes and timing of GnRHa combined with hCG trigger, for final follicular maturation, have been described [2].

A modification of the "dual trigger," the so-called double trigger approach, has been successfully used in a patient with recurrent empty follicle syndrome [3]. The "double trigger," which consists of the co-administration of GnRH-agonist and hCG for final oocyte maturation, 40 and $34 \mathrm{~h}$ prior to ovum pick-up (OPU), respectively, differs from the "dual trigger" by the additional prolongation of the time between ovulation triggering and OPU. This later prolongation may explain the beneficial effect in terms of both oocytes yield/maturation and pregnancy rate.

Raoul Orvieto

raoul.orvieto@sheba.health.gov.il

1 Infertility and IVF Unit, Department of Obstetrics and Gynecology, Chaim Sheba Medical Center (Tel Hashomer), Ramat Gan and the Tarnesby-Tarnowski Chair for Family Planning and Fertility Regulation, Sackler Faculty of Medicine, Tel Aviv University, Tel Aviv, Israel
The "double trigger" was also shown to significantly improve the oocyte yield in patients with low/poor oocytes yield, despite an apparently normal follicular development and E2 levels on the day of hCG administration [4] and increase the proportions of metaphase-II (MII) oocytes in patients with low proportion of mature oocytes per number oocytes retrieved [5].

We recently suggested to tailor final-follicularmaturation trigger [6]. We offer standard hCG dose concomitant with GnRHa ("dual trigger") 35-37 h before oocyte retrieval to normal-responder patients, aiming to improve oocytes yield, number of matured oocytes, and top-quality and cryopreserved embryos. GnRHa $40 \mathrm{~h}$ and standard hCG added $34 \mathrm{~h}$ prior to OPU (double trigger), respectively, are offered to two groups of patients demonstrating abnormal final follicular maturation despite normal response to $\mathrm{COH}$, those with low $(<50 \%)$ number of oocytes retrieved per number of dominant follicles $>14 \mathrm{~mm}$ in diameter on the day of hCG administration and those with low proportion of mature/ metaphase-II (MII) oocytes $(<66 \%)$ per number oocytes retrieved. Finally, standard hCG dose concomitant with GnRHa ("dual trigger"), 34 h before oocyte retrieval was offered to poor-responder patients, aiming to improve oocytes yield and maturation and prevent premature luteinization.

\section{References}

1. Elias RT, Pereira N, Artusa L, Kelly AG, Pasternak M, Lekovich JP, et al. Combined GnRH-agonist and human chorionic gonadotropin trigger improves ICSI cycle outcomes in patients with history of poor fertilization. J Assist Reprod Genet. 2017;34(6):781-8.

2. Orvieto R. Triggering final follicular maturation-hCG, GnRHagonist or both, when and to whom? J Ovarian Res. 2015;8:60. 
3. Beck-Fruchter R, Weiss A, Lavee M, et al. Empty follicle syndrome: successful treatment in a recurrent case and review of the literature. Hum Reprod. 2012;27:1357-67.

4. Haas J, Zilberberg E, Dar S, Kedem A, Machtinger R, Orvieto R. Coadministration of GnRH-agonist and hCG for final oocyte maturation (double trigger) in patients with low number of oocytes retrieved per number of preovulatory follicles-a preliminary report. J Ovarian Res. 2014;7:77.
5. Zilberberg E, Haas J, Dar S, Kedem A, Machtinger R, Orvieto R. Coadministration of GnRH-agonist and hCG for final oocyte maturation in patients with low proportion of mature oocytes. Gynecol Endocrinol. 2015;31:145-7.

6. Orvieto R. A simplified universal approach to $\mathrm{COH}$ protocol for IVF: ultrashort flare GnRH-agonist/GnRH-antagonist protocol with tailored mode and timing of final follicular maturation. J Ovarian Res. 2015;8:69. 